Presented at the 1992 Intl. Conf. of

Applications of Particle Accelerators,

Denton, TX - October 1992

\title{
FABRICATION AND TEST OF A SUPERCONDUCTING RFQ*
}

A. JAIN(a), H. WANG(a), 1. BEN-ZVI(a), P. PAUL and J.W. NOÉ

DE93 010860

Department of Physics, SUNY at Stony Brook, NY 11794-3800 USA

A. LOMBARDI

INFN-LNL, Via Romea 4, Legnaro (PD) I-35020, Italy

The fabrication and first performance tests of a prototype superconducting radio-frequency quadrupole resonator (SRFQ) are described. The SRFQ operates at $57 \mathrm{MHz}$ and is optimized for a particle velocity of $\beta=0.033$. It is constructed of copper electroplated with a lead-tin alloy. An accelerating field gradient of $1.25 \mathrm{MV} / \mathrm{m}$ was achieved with about 7 watts of helium dissipation. This corresponds to an energy gain of $700 \mathrm{keV}$ per unit charge over the $56 \mathrm{~cm}$ overall diameter of the resonator.

\section{Introduction}

A superconducting radio-frequency quadrupole (SRFQ) would be very useful for the continuous (cw) acceleration of low-velocity heavy-ion beams since it would combine the efficient acceleration and intrinsic focussing of the RFQ with very modest power dissipation. A recent paper [1] has described an SRFQ pre-accelerator for boosting ions as heavy as $\mathrm{Pb}$ from an ECR source up to $\beta \sim 0.05$ for injection into a superconducting linac. The proposed design breaks the long classical RFQ into a series of compact independent elements each optimized (by proper choice of modulation parameter $m$ and aperture a) for maximum acceleration per unit surface field over a particular velocity range.

The present paper describes the fabrication and first superconducting tests of the third of six elements described in Ref.[1]. Further details of the SRFQ design and fabrication can be found in other conference papers $[2,3]$ and a thesis $[4]$.

\section{Description}

The prototype SRFQ resonator is shown in Fig. 1. The active elements of the structure are the four steeply modulated $(m=4) 40 \mathrm{~cm}$ long vanes surrounding the beam axis (minimum aperture radius $a=15.7 \mathrm{~mm}$ ). The two pairs of diagonally opposed vanes of the same electrical polarity are interconnected through four curved electrode support tubes ( $4 \mathrm{~cm}$ dia.) and two spheres (12 $\mathrm{cm}$ dia.). These two vane subassemblies are in turn suspended from the reinforced top plate of the outer container by two large ( $8 \mathrm{~cm} \mathrm{dia.)} \mathrm{hang-}$

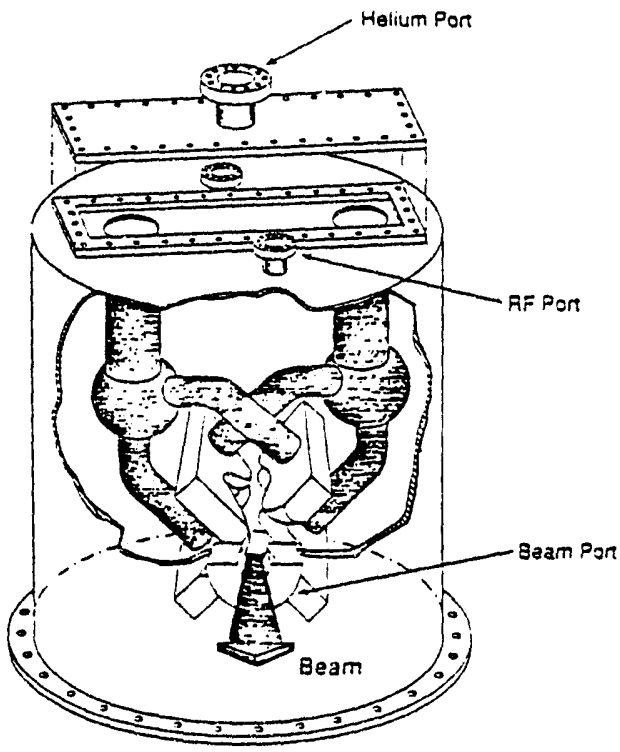

Figure 1: Cut-away view of the prototype SRFQ. The overall size is about $0.6 \times 0.6 \mathrm{~m}$.

ing tubes. Beam enters and leaves the resonator through large beam ports with $32 \mathrm{~mm}$ openings. The two small extended ports on the top plate are for the rf input and pickup loops.

The design goal accelerating field of the prototype is $E_{n}=2 \mathrm{MV} / \mathrm{m}$. The $E_{n}$ used here is defined in terms of the energy gain $\Delta W$ of a particle with charge $q$ by $\Delta W=E_{a} \cdot L \cdot T(\beta)$ where $T\left(\beta_{n}=0.033\right)=1.0$ and $L=55.8 \mathrm{~cm}$ is the total insertion length of the resonator from the outside face of one beam port to the other. Relevant electrical parameters at the $2 \mathrm{MV} / \mathrm{m}$ design field are:

*This work was performed under the auspices of the U.S. Dept. of Energy. 
energy content $U=3.9$ joules, intervane voltage $=420 \mathrm{kV}$, peak $E_{1} \simeq 15 \mathrm{MV} / \mathrm{m}$, and peak $B_{n} \simeq$ $360 \mathrm{G}$ [2]. The $E$, and $B$, maxima occur at the vane tips and near the roots of the hanging tubes, respectively. The $57 \mathrm{MHz}$ resonant frequency is $\sim 15 \%$ higher than the nominal value [1] because of size constraints imposed by the test cryostat.

The SRFQ resonator is made from copper electroplated with a lead-tin alloy superconductor. The micron thick alloy films were plated directly with no subsequent chemical polishing step. This "thinlead" technology has proven quite successful in other low-frequency heavy-ion linac resonators $[5,6]$ and a large cyclotron cavity [7]. For optimum cooling by the liquid helium bath the entire electrode structure is hollow and the supporting tubes are shaped to allow for the free escape of gas bubbles.

The prototype SRFQ was plated and subsequently tested at $4.2 \mathrm{~K}$ three times. In the first two tests the $Q$ reached an acceptable value of $10^{8}$ only at extremely low fields $(0.01 \mathrm{MV} / \mathrm{m})$. A greatly improved $Q$ curve was obtained in the third test, after adjusting the plating parameters and reworking the copper surface of the top plate to remove voids and braze blobs that might have limited thermal conductivity.

\section{Construction}

The various parts of the SRFQ structure were fabricated by machining and spinning operations and assembled mainly by furnace brazing. OFHC (CDA 101) is the preferred grade of copper because of its high thermal conductivity and high purity, but it was not practical to use this material throughout. Electrolytic tough pitch copper (CDA 110) was chosen for the electrodes (vanes), bottom cover and helium manifold. The vanes were carefully shaped and hollowed out on a CNC milling machine at Stony Brook. Vane covers were machined separately. The spheres were made from two spun OFHC half-spheres and the beam ports were turned from OFHC blocks. The outer vessel proved to be too large to be fabricated from a single spinning. Therefore a shallow OFHC spinning for the top cover was joined to a rolled OFHC outer wall by TIG welding. Finally, the hanging and electrode support tubes were made from various commercial phosphorous de-oxidized copper pieces.
Assembiy of the structure started with e-beam welding to tack the vane covers in place and assemble the spheres and electrode support tubes from component parts. The remaining assembly was done in three eycles of furnace brazing under argon purge. (Hydrogen could not be used because not all parts are OFHC copper.) In the first pass the vane covers were secured with Nicusil3 alloy wire (liquidus $=795^{\circ} \mathrm{C}$ ). Because alloying with the copper raises the liquidus temperature this vane joint could be safely brought to the same temperature for a second Nicusil-3 braze cycle to complete the two electrode pair subassemblies. In the final assembly braze the two electrode subassemblies and the remaining parts (beam ports, RF ports, helium manifold, hanging tabs) were joined to the external can using Incusil-15 wire and powder (liquidus $=705^{\circ} \mathrm{C}$ ). Parts were secured in accurate relative positions during brazing with various 304 stainless steel fixtures. After the final braze, some cracks were found in the beam port joints. These were filled with soft solder to avoid trapping of acid solutions. The completed resonator is quite heavy $(100 \mathrm{~kg})$ in relation to its strength and hence not easily handled safely. This was solved by bolting a large $U$ bracket to the helium manifold on the top plate to provide pivot points close to the overall center of mass.

\section{Electroplating}

The resonator was electroplated mouth-up using the can as its own container. To achieve complete coverage a $10 \mathrm{~cm}$ extension collar was strapped to the outer lip of the can and temporarily sealed with a silicone adhesive compound. The cover plate was plated separately and later attached with a 50-50 tin-indium wire gasket.

The plating anodes were two cylinders and two L-shaped pieces formed from lead sheet and enclosed in dyrel bags. A low current density of 0.25 $\mathrm{mA} / \mathrm{cm}^{2}$ was used with periodic reversal ( $8 \mathrm{~s}$ forward, then $2 \mathrm{~s}$ reverse). The uniformity was tested in a scale model and found to be within $\pm 15 \%$. For the third plating the average film thickness was reduced from $5.0 \mu \mathrm{m}$ to $1.5 \mu \mathrm{m}$ to achieve a better surface texture. The plating bath was initially the same standard 93:7 commercial fluoborate preparation previously used to replate Stony Brook linac modules [8] and retrofit QWRs [9]. For the final plating this was changed to a new 


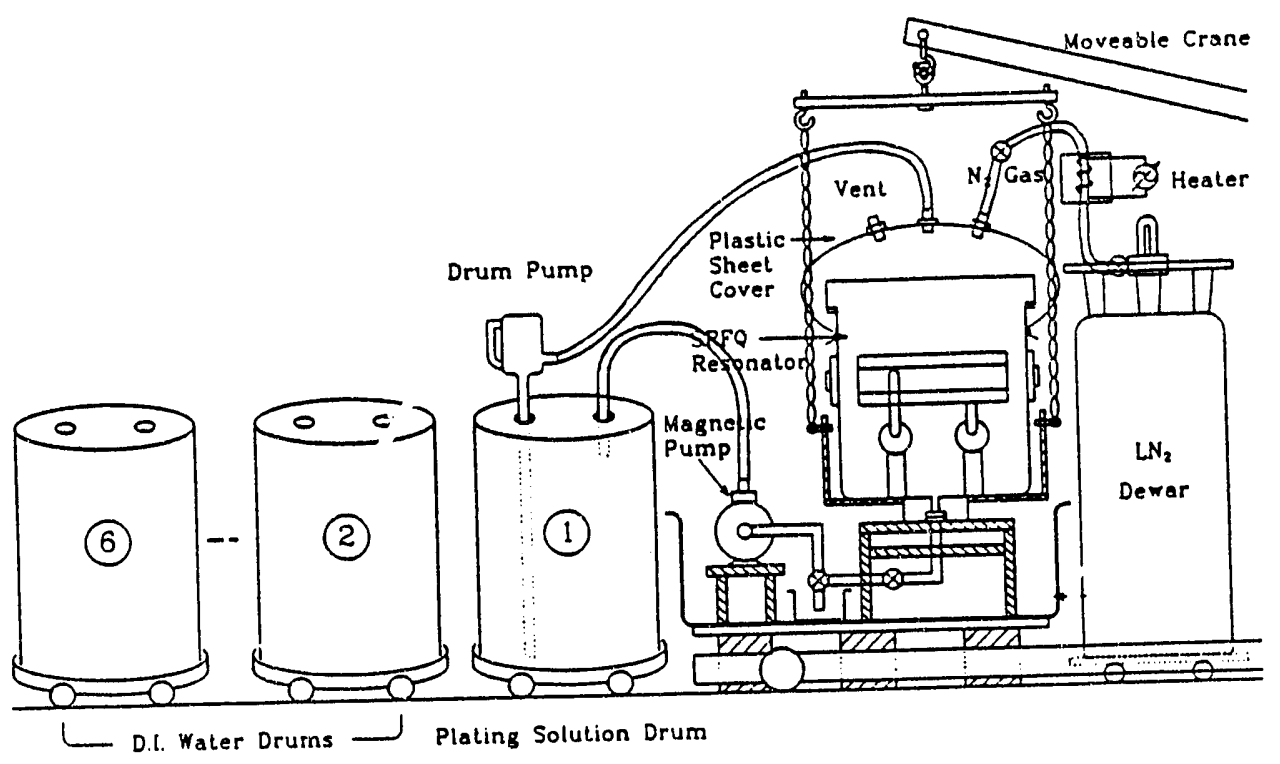

Figure 2: Scheme for rinsing the SRFQ after plating in a nitrogen gas atmosphere.

bath with only 2 at. $\%$ tin ( $1 \%$ by weight) to avoid any possible precipitation of the tin component of the alloy [4]. The bath was filtered for 8 hours before use and heated to $35 \mathrm{C}$. It was also filtered continuously during the plating.

Figure 2 shows the simple but very effective system used to prevent staining and oxidation of the plated surfaces during the lengthy (30 minutes) rinsing and drying steps. This scheme has since been adapted to the smaller $Q W R$ with excellent results [5]. After the plating the anodes were lifted out of the bath and a clear flexible plastic sheet dome attached (Fig. 2). The dome was continuously purged with boiloff nitrogen gas as the bath was pumped out and the plated surface vigorously rinsed with $\sim 1000$ l of de-ionized water. The flexibility of the dome allowed the rinse water to be directed in turn against every area of the structure. After the rinse the surface was allowed to dry without solvents.

\section{Superconducting tests}

The cold tests were carried out in the same offline test cryostat used for the development of the superconducting quarter-wave resonator ( $Q W R$ ), with similar procedures [10]. Prior to cooldown the SRFQ was baked for one day at $50 \mathrm{C}$ with the liquid nitrogen shield cold. Since there was no space in the cryosiat for a separate precool line the initial phase of the cooldown had to be done by filling the SRFQ with liquid nitrogen. Considerable care was then required to ensure that no nitrogen remained in the electrode structure after reaching $77 \mathrm{~K}$. The helium phase of the cooldown consumed $\sim 150$ liquid liters but otherwise presented no special problems. Multipactor conditioning was done at room temperature with a $1.5 \mathrm{~kW}$ pulse amplifier. A very wide range of levels was observed but all could be conditioned away in about 48 hours.

Figure 3 shows the $Q$-curve from the third plating and test cycle. The low-field $Q$ is $1.5 \cdot 10^{8}$ and $Q$ remains above $10^{8}$ up to $E_{a}=1 \mathrm{MV} / \mathrm{m}$. The dissipation at $1 \mathrm{MV} / \mathrm{m}$ is only about 3 watts. Suddenly at about $1.3 \mathrm{MV} / \mathrm{m}$ however the $Q$ drops very quickly and this barrier cannot be passed even with short pulses. The limitation appears in the pick-up signal as a soft collapse to a stable lower field level after some 10 milliseconds. Several hours of high-power pulse conditioning both with and without helium gas did not significantly extend the achievable field. The helium conditioning did however significantly reduce $x$-ray production and slightly extend the $Q$ curve. These observations suggest that the limitation at $1.3 \mathrm{MV} / \mathrm{m}$ is 


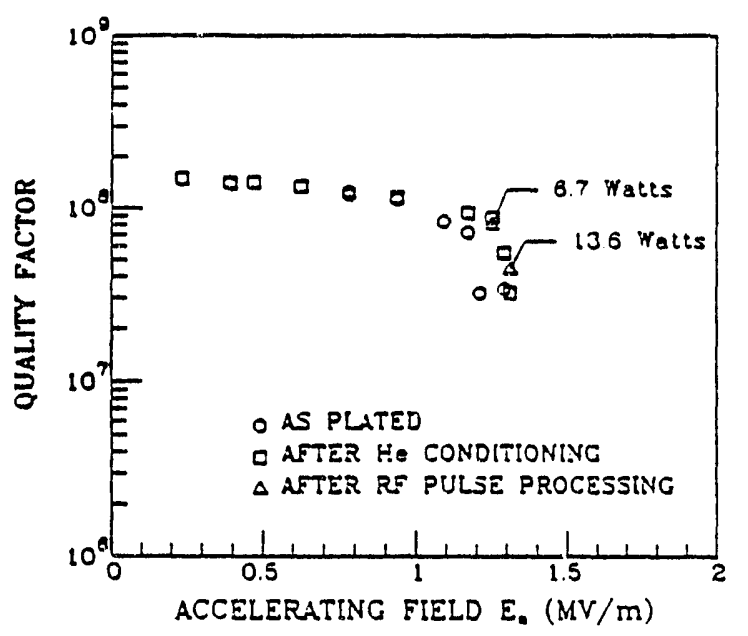

Figure 3: $Q$ curve from the SRFQ cold test.

not related to field emission but rather most likely to an excessive magnetic field in a high-current region or to inadequate local cooling from some remaining imperfections in the copper.

No attempt was made in this test to tune or frequency stabilize the SRFQ, but some measurements were made of the eigenfrequency excursions to guide future developments. These were about $60 \mathrm{~Hz}$ peak-to-peak with a very noisy cryostat pump running and about $20 \mathrm{~Hz}$ with the pump turned off. These figures are reasonable for such a large and complex structure.

\section{Conclusions}

The present work has shown that it is feasible to build and operate a practical SRFQ device with standard laboratory techniques. Multipactoring is easily eliminated by routine if pulse processing, and vibration appears to be manageable. While the acceleration of $\sim 700 \mathrm{ke} b$ per charge so far achieved falls somewhat short of the $2 \mathrm{MV} / \mathrm{m}$ design goal it should be more than sufficient to begin the critical beam testing phase of the development program.

\section{Acknowledgements}

We wish to thank the staff of the Physics Department machine shop and J. Rico and H. Uto of the QWR group for their many contributions to the resonator fabrication and plating effort. The SRFQ development was supported in part by NSF Grant No. PHY-8902923.

\section{References}

[a] Now at Brookhaven National Laboratory, Upton, New York 11973.

[1] I. Ben-Zvi, A. Lombardi and P. Paul, Part. Accel. 35 (1991) 177.

[2] A. Jain, I. Ben-Zvi, P. Paul, H. Wang and A. Lombardi, Proc. 1991 Part. Accel. Conf., IEEE Conf. Record 91CH3038-7, 2444.

[3] I. Ben-Zri, A. Jain, J.W. Noé, P. Paul, H. Wang, and A. Lombardi, 12 th Int. Conf. on Applications of Accelerators in Research and Indusiry, Denton, TX, November 2-5, 1992.

[4] Haipeng Wang, Master of Science in Instrumentation thesis, SUNY at Stony Brook, 1992.

[5] J.W. Noé, J. Rico and H. Uto, this conference.

[0] J.R. Delayen and J.E. Mercereau, Nucl. Instr. and Methods A257 (1987) 71.

[7] L. Dietl and U. Trinks, Nucl. Instr. and Methods A284 (1989) 293.

[8] J. Sikora, I. Ben-Zvi, J.M. Brennan, M. Cole and J.W. Noe, Third Workshop on Rf Superconductivity. Argonne National Laboratory (1987).

[9] J.W. Noé, R. Arora, J. Rico, J. Sikora and H. Uto, Nucl. Instr. and Methods A287 (1990) 240.

[10] J.M. Brennan, B. Kurup, I. Ben-Zvi and J.S. Sokolowsky, Nucl. Instr. and Meth. A242 (1985) 23. 

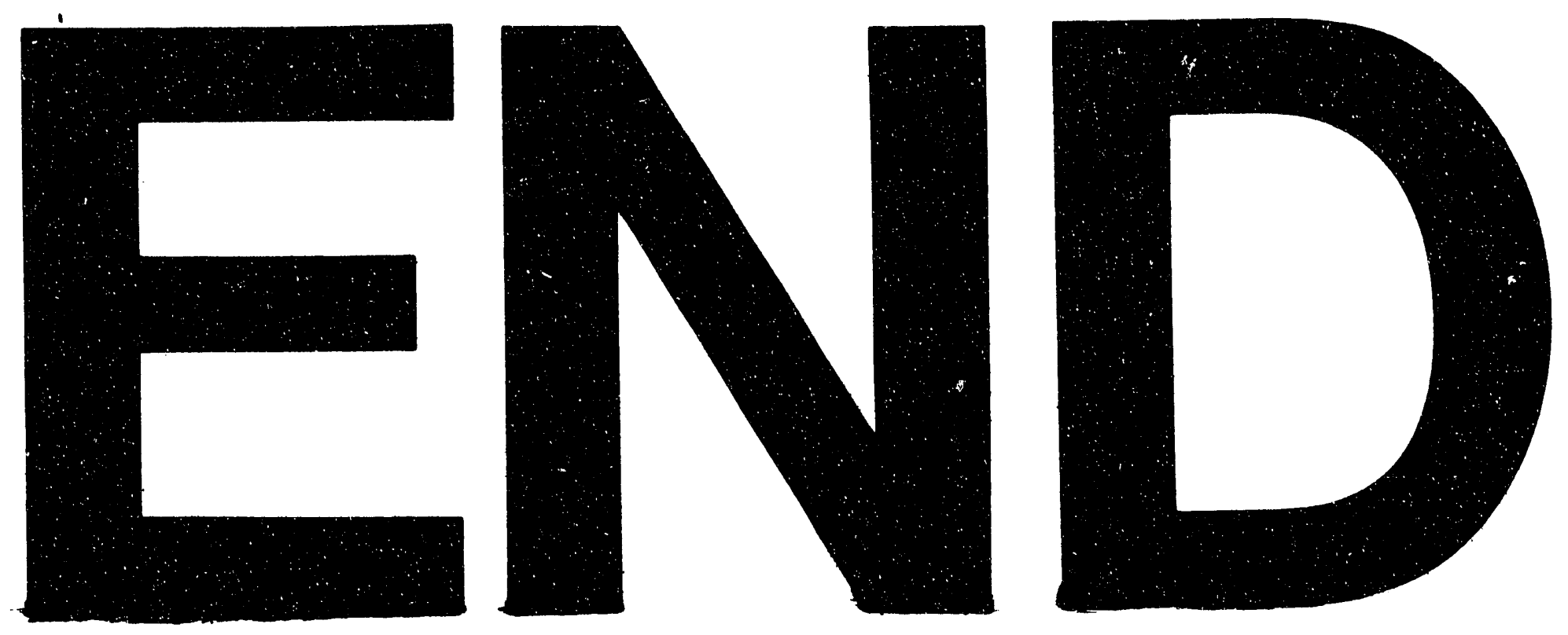

18
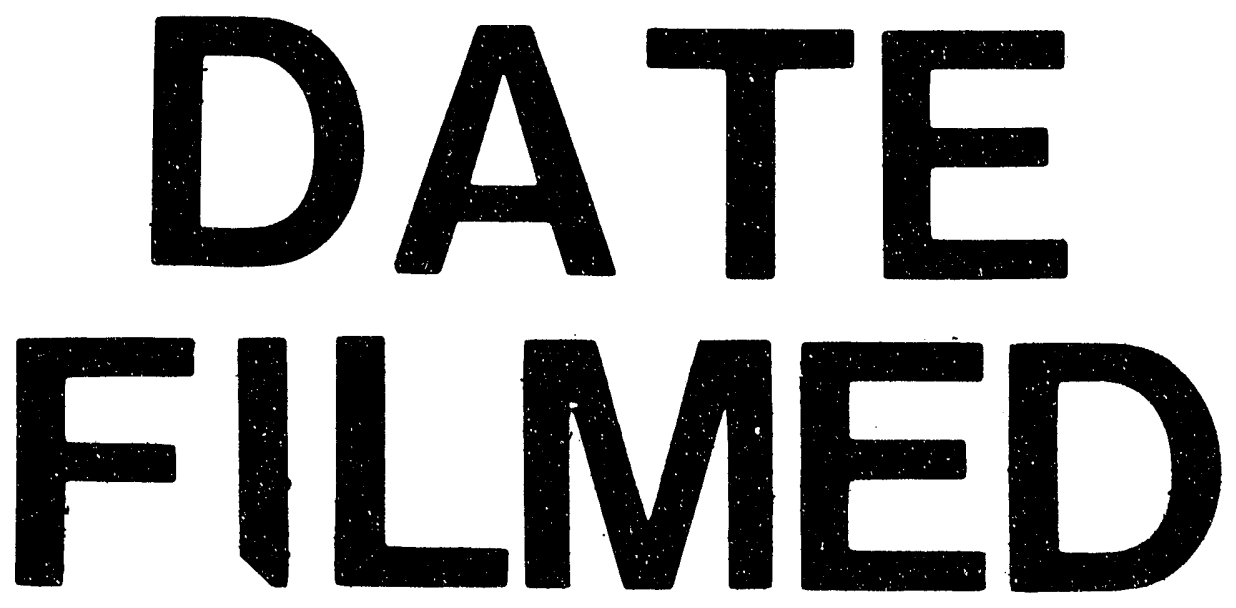

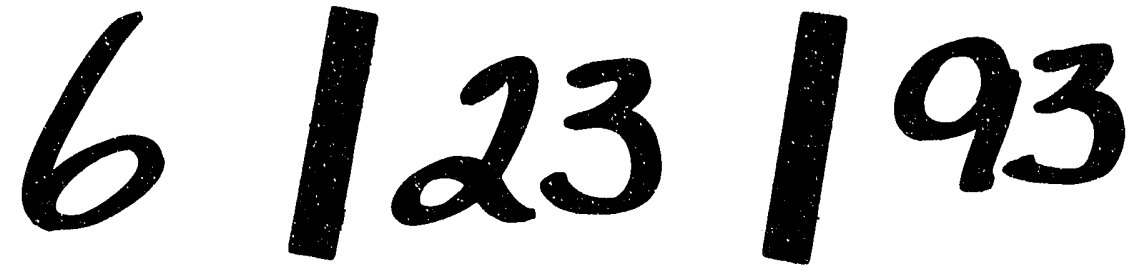


$\|111 \quad\| 1\|1, \quad\| 1$

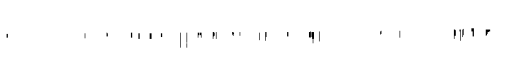

